\title{
Camera-trap surveys reveal high diversity of mammals and pheasants in Medog, Tibet-CORRIGENDUM
}

DOI: 10.1017/S0030605319001467. Published online by Cambridge University Press, 14 April 2020

The order of species names on the y-axis was incorrect in Fig. 2 of this Short Communication. The correct figure is provided here.

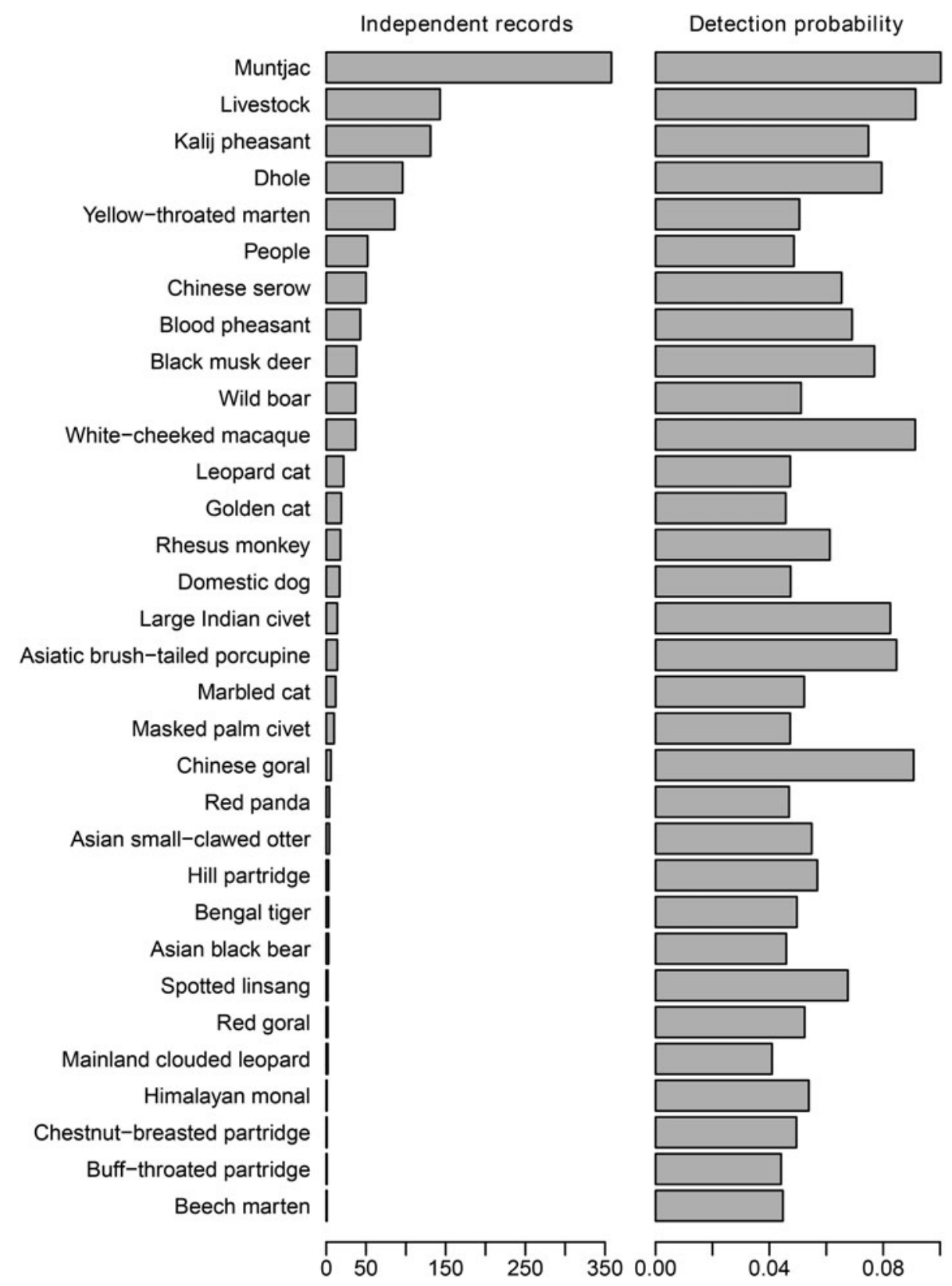

\section{Reference}

Li, X., Bleisch, W.V., LiU, X. \& JiAng, X. (2020) Camera-trap surveys reveal high diversity of mammals and pheasants in Medog, Tibet. Oryx, published online 14 April 2020.

FIG. 2 Number of independent records, mean individual detection probability and mean occupancy for the 23 medium and large terrestrial mammal species, six pheasant species, and livestock, people and domestic dogs in the camera-trap surveys in Medog County (Fig. 1). 